\title{
Modulation of P-glycoprotein expression by triptolide in adriamycin-resistant K562/A02 cells
}

\author{
HAO LI, LULU HUI, WENLIN XU, HUILING SHEN, QIAOYUN CHEN, LULU LONG and XIAOLAN ZHU \\ Department of Central Laboratory, The Affiliated People's Hospital, Jiangsu University, Jiangsu, P.R. China
}

Received October 3, 2011; Accepted November 25, 2011

DOI: $10.3892 / \mathrm{ol} .2011 .500$

\begin{abstract}
Multidrug resistance is a serious obstacle encountered in leukemia treatment. Previous studies have found drug resistance in human leukemia is mainly associated with overexpression of the multidrug resistance gene 1 (MDR1). The aim of the present study was to investigate the modulation of $\mathrm{P}$-glycoprotein expression by triptolide in adriamycin-resistant K562/A02 cells. The reverse effects of triptolide on drug resistance in K562/A02 cells were assessed by 3-[4,5-dimethylthiazol-2-yl]-2, 5-diphenyl-tetrazolium bromide (MTT) assay. The percentage of apoptotic cells was obtained from annexin V/fluorescein isothiocyanate (FITC) and propridium iodide (PI) double-staining. The effects of triptolide on P-glycoprotein activity were evaluated by measuring intracellular adriamycin accumulation. The expression of P-glycoprotein was determined by flow cytometry. A luciferase reporter gene assay was used to detect the transcriptional activity of the MDR 1 promoter. Results revealed that triptolide decreased the degree of resistance of K562/A02 cells, and significantly inhibited P-glycoprotein expression and drug-transport function, and increased the accumulation of adriamycin in K562/A02 cells as measured by flow cytometry. A luciferase reporter gene assay demonstrated that triptolide was capable of inhibiting the transcriptional activity of the MDR1 promoter. Triptolide may effectively reverse the adriamycin resistance in K562/A02 cells via modulation of the P-glycoprotein expression and by increasing intracellular adriamycin accumulation.
\end{abstract}

\section{Introduction}

Chemotherapy is used systematically to treat patients with leukemia. Failure of the radical cure of leukemia patients often occurs as a result of intrinsic or acquired drug resistance of the

Correspondence to: Professor Wenlin $\mathrm{Xu}$, Department of Central Laboratory, The Affiliated People's Hospital, 8 Dianli Road, Zhenjiang, Jiangsu 212001, P.R. China

E-mail: xuw10511@yahoo.com

Key words: triptolide, multidrug resistance, apoptosis, P-glycoprotein leukemia cells to chemotherapeutic agents. Although the exact mechanism remains unclear, the role of ATP-dependent drug efflux-proteins on the cell membrane is currently accepted as a major cause behind cancer drug resistance. The P-glycoprotein transporter is a $170-\mathrm{kD}$ plasma membrane glycoprotein encoded by the human multidrug resistance gene 1 (MDR1). $\mathrm{P}$-glycoprotein is capable of transporting intracellular drugs out of cells to acquire drug resistance (1-3). A number of natural and synthetic substances have been tested for their ability to overcome MDR in vitro and in vivo (4). These substances include calcium channel blockers (e.g., verapamil), calmodulin antagonists (e.g., trifluoperazine) and numerous polyphenolic compounds, including curcuminoids, curcumin and eigallocatechin gallate (EGCG) (5-7). However, none of these compounds have been applied in the clinic owing to their severe side effects and poor pharmacokinetics in vivo (8).

Triptolide (TPL), a diterpenoid triepoxide derived from Tripterygium wilfordii Hook f (TWHf; also known as Lei Gong Teng), has been used as a natural therapeutic agent in traditional Chinese medicine for centuries. TPL has been used successfully in clinical treatment of rheumatoid arthritis, autoimmune diseases (e.g., rheumatoid arthritis, nephritic syndrome, ulcerative colitis, asthma, idiopathic pulmonary fibrosis) and organ transplant anti-rejection (9). In recent years, TPL has also demonstrated strong anti-proliferative activity and induction of apoptosis in a broad range of cancers in vitro and in vivo. TPL induces apoptosis in a variety of leukemic cell lines and primary acute myeloid leukemia blasts via the mitochondrial pathway (10). Clinical trials in China have demonstrated TPL-induced remission rates of $71-87 \%$ in mononucleocytic and granulocytic leukemia (11). TPL and its water-soluble derivative TPL-88 also demonstrate enhancing effects on the cytotoxicity of certain cytokines and conventional anti-cancer drugs $(10,12-14)$. Numerous studies reveal that TPL is a strong inhibitor of NF- $\kappa \mathrm{B}$, which is capable of regulating the expression of the MDR1 gene. Therefore, TPL may modulate $\mathrm{P}$-glycoprotein expression through inhibition of $\mathrm{NF}-\kappa \mathrm{B}$ activity.

In the present study, we observed the enhancing effect of TPL on the cytotoxicity of adriamycin in MDR leukemia cell line K562/A02 and intended to explore the potential molecular chemosensitizing mechanisms. Our results demonstrate that TPL strongly enhanced the cytotoxicity and apoptosis of adriamycin through downregulation of P-glycoprotein in adriamycin-resistant K562/A02 cells. 


\section{Materials and methods}

Cell lines and cell culture. The human chronic myeloid leukemia cell line K562, and its MDR counterpart K562/A02, were purchased from the Shanghai Institute of Cell Biology, Chinese Academy of Sciences (China). K562 cells were cultured in complete RPMI- 1640 at $37^{\circ} \mathrm{C}$ in a humidified atmosphere of $5 \% \mathrm{CO}_{2}$. K562/A02 cells were cultured in a medium containing $1 \mu \mathrm{g} / \mathrm{ml}$ adriamycin for maintaining the MDR phenotype. The cells were passaged every 2-3 days and cultured for 2 weeks in drug-free medium prior to their use in the experiments.

Cytotoxicity assay. Cell viability was assessed by 3-[4,5-dimethylthiazol-2-yl]-2, 5-diphenyl-tetrazolium bromide (MTT) assay as reported previously (15). Cells were seeded into 96-well plates in RPMI-1640 containing 10\% fetal bovine serum (FBS). The cells were treated with serial dilutions of TPL. Approximately $72 \mathrm{~h}$ following TPL treatment, MTT was added at a final concentration of $0.5 \mathrm{mg} / \mathrm{ml}$, and the cells were incubated for $4 \mathrm{~h}$ at $37^{\circ} \mathrm{C}$. The optical density was read at $570 \mathrm{~nm}$ with a microplate spectrophotometer. Each experiment was repeated in triplicate.

Drug resistance reversal assay. K562/A02 cells seeded into 96-well plates were treated with various concentrations of adriamycin in the absence and presence of TPL at doses of $5 \mathrm{nmol} / \mathrm{l}$ for $72 \mathrm{~h}$, respectively. By using the same MTT assay as mentioned above, the inhibition ratio of each adriamycin concentration with or without TPL was calculated using the Statistical Package for Social Science (SPSS) method. In all the experiments, each group was conducted in 6 individual wells.

Apoptosis assay. Annexin V/fluorescein isothiocyanate (FITC) and propidium iodide (PI) apoptosis detection kit (BectonDickinson, Franklin Lakes, NJ, USA) was used to quantitatively measure the phosphatidylserine in apoptotic cells. Briefly, cells $\left(5 \times 10^{5}\right.$ per well) were seeded into 6 -well plates and then treated with $3 \mu \mathrm{g} / \mathrm{ml}$ of adriamycin in the absence and presence of 5 $\mathrm{nmol} / \mathrm{l}$ TPL. After $24 \mathrm{~h}$, the cells were harvested and washed twice with ice-cold phosphate-buffered saline (PBS) (pH 7.2). After $5 \mathrm{~min}$ of centrifugation at $200 \mathrm{x}$ g, Annexin V/FITC and PI double-staining were performed according to the manufacturer's instructions. Cell apoptosis was analyzed on a FACScan flow cytometry (Becton-Dickinson). Annexin V-positive, PI-negative cells were scored as apoptotic. Double-stained cells were considered to be necrotic or late apoptotic.

Intracellular adriamycin accumulation assay. K562 and $\mathrm{K} 562 / \mathrm{A} 02$ cells were plated in a 6-well plate at a concentration of $5 \times 10^{5}$ cells in $2 \mathrm{ml}$ of growth medium. After $24 \mathrm{~h}$, TPL was added to the K562/A02 cells for a further $24 \mathrm{~h}$. K562/A02 cells were incubated with $3 \mu \mathrm{g} / \mathrm{ml}$ adriamycin (with or without TPL) for $1 \mathrm{~h}$ at $37^{\circ} \mathrm{C}$. Cells were harvested following three washes with ice-cold PBS. The cells were subsequently subjected to flow cytometry (FACSAria; Becton-Dickinson). The K562 cell was used as a positive control for maximum adriamycin accumulation.

P-glycoprotein expression assay. The cell-surface P-glycoprotein levels were measured by flow cytometry $(16,17)$. K562/A02 cells seeded into 24 -well plates at a density of $1 \times 10^{5}$

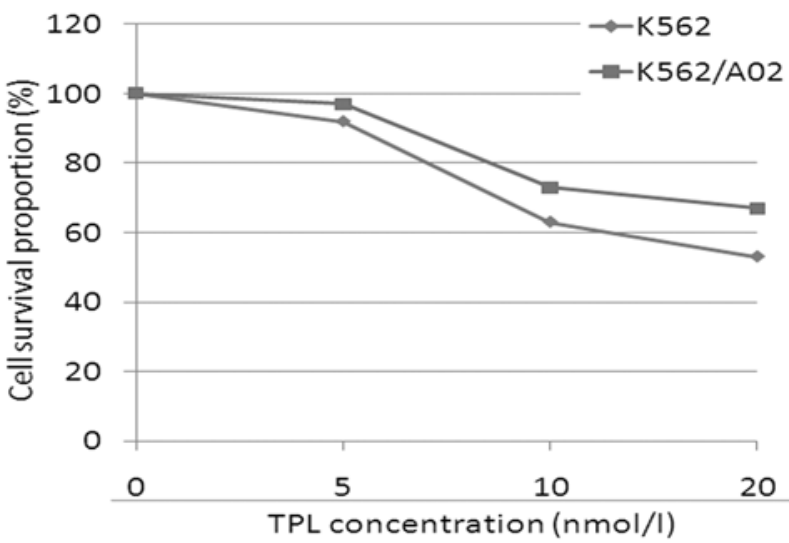

Figure 1. Effects of TPL on the growth of K562/A02 cells. The cells were treated with various concentrations of TPL for $72 \mathrm{~h}$, respectively. Viable cells were evaluated by MTT assay. MTT, 3-[4,5-dimethylthiazol-2-yl]-2, 5-diphenyl-tetrazolium bromide; TPL, triptolide.

per well were treated with $5 \mathrm{nmol} / 1 \mathrm{TPL}$ for $24 \mathrm{~h}$, respectively. The cells were harvested, washed twice with ice-cold PBS, counted and then labeled with mouse anti-human monoclonal antibody against P-glycoprotein according to the manufacturer's instructions. The fluorescent intensity was analyzed using FACSCalibur with isotype as a control. Duplicate experiments with triplicate samples were performed.

Luciferase reporter gene assay. Construction of the MDR1 promoter reporter plasmid and luciferase assay were described in a previous study (18). To obtain the MDR1 promoter fragments, polymerase chain reaction (PCR) amplification on human genomic DNA (forward primer 5'-GGG GTACCCCAGTCTCTACG-3', reverse primer 5'-CAAGCTT GTCCGACCTGAAGAG-3') was performed in a $50 \mu 1$ reaction mixture. The PCR product was cloned into the KpnI/HindIII site of the pGL3-basic vector. K562/A02 cells were plated in 6-well plates at a density of $5 \times 10^{5}$. pGL3-MDR1-promoter plasmid $(2 \mu \mathrm{g})$ was transfected using the Lipofectamine 2000 (Invitrogen, Carlsbad, CA, USA) according to the manufacturer's instructions. Following transfection (4-6 h), the cells were reseeded in 96-well plates and TPL was added to the cells at a final concentration of $5 \mathrm{nmol} / \mathrm{l}$. After $24 \mathrm{~h}$, the cells were harvested with extraction buffer $(25 \mathrm{mM}$ glycylglycine, pH 7.8, $15 \mathrm{mM} \mathrm{MgSO}_{4}, 4$ mM EGTA and 1\% Triton X-100). Luciferase activity was measured using a FLUOstar OPTIMA system (BMG LABTECH GmbH, Ortenberg, Germany). Transfection experiments were repeated at least three times.

Statistical analysis. Experiments were repeated at least three times; data are presented as the mean \pm standard deviation (SD) and were analyzed using the SPSS method (SPSS Release 12.0; SPSS Inc., Chicago, IL, USA). Student's t-test was used to assess the statistical significance of differences. $\mathrm{P}<0.05$ was considered to indicate a statistically significant difference.

\section{Results}

Cytotoxicity of TPL. To investigate the effect of TPL on MDR, human leukemia cell lines K562 and K562/A02 were used to 
A

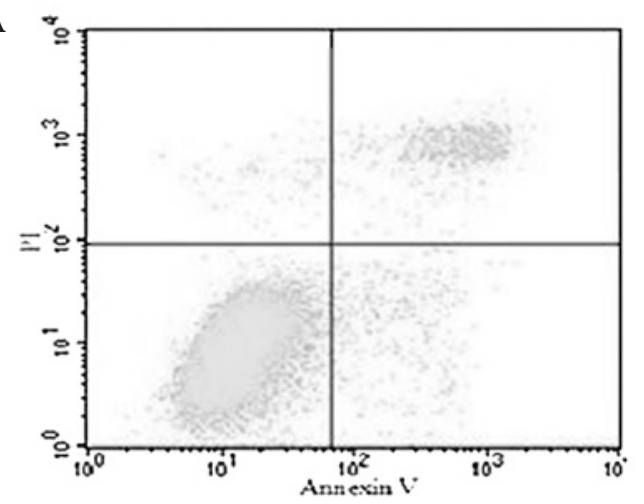

C

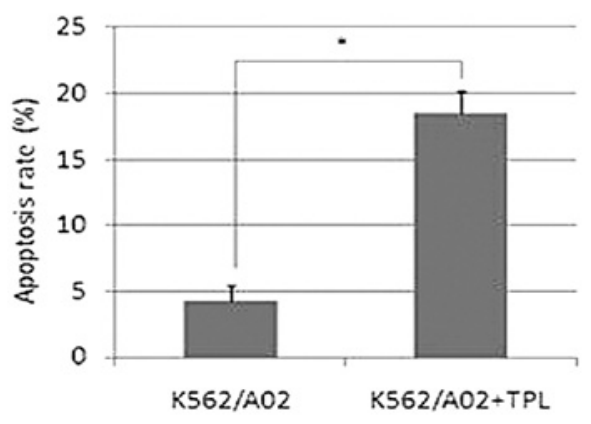

B
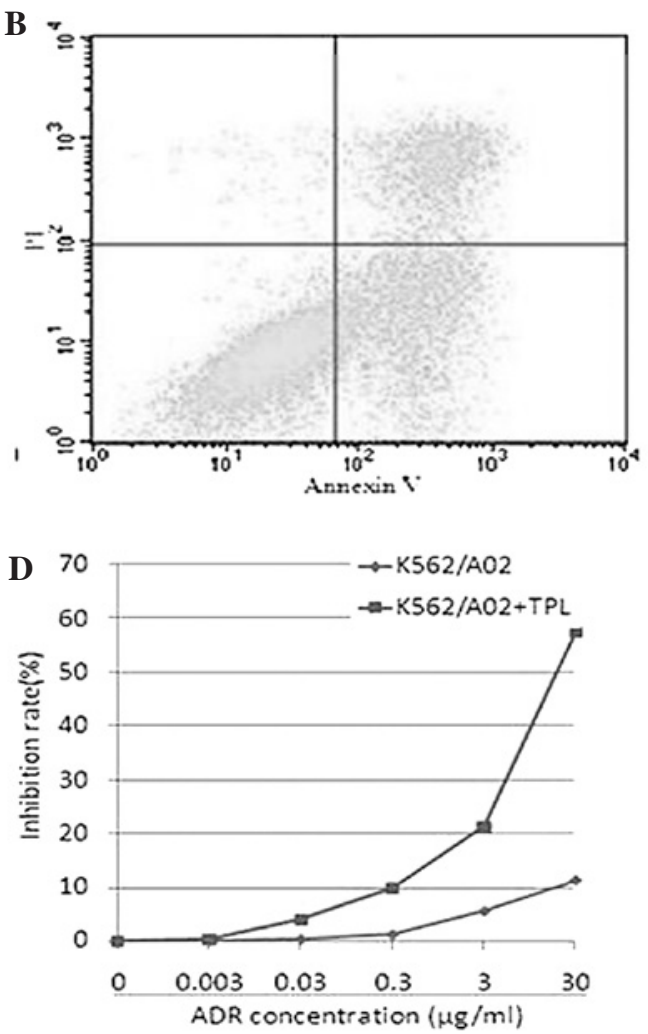

Figure 2. Reversal of adriamycin resistance in vitro by TPL. K562/A02 cells were incubated with $3 \mu \mathrm{g} / \mathrm{ml}$ of adriamycin in the presence of, or without, TPL for $24 \mathrm{~h}$. The rates of apoptosis were measured using flow cytometry. The cell inhibition rate was measured by MTT. (A) Apoptosis in K562/A02 cells; (B) apoptosis in K562/A02 treated with TPL; (C) apoptosis rate; (D) inhibition rate. MTT, 3-[4, 5-dimethylthiazol-2-yl]-2, 5-diphenyl-tetrazolium bromide; TPL, triptolide; ADR, adriamycin; PI, propridium iodide.

determine the non-toxic dose of TPL. The cytotoxic effects of TPL on K562 and K562/A02 cells were measured following a $72 \mathrm{~h}$ treatment (Fig. 1), since the reversal multidrug agents are required to be maintained at appropriate concentrations, at which the drugs have no inhibitory or toxic effects on the tested cells. Consequently, a concentration of $5 \mathrm{nmol} / \mathrm{l} \mathrm{TPL}$ was used to study the reversal effect of MDR.

Reversal of adriamycin resistance in vitro by TPL. To investigate the effect of TPL on the sensitivity of K562/A02 cells to a chemotherapeutic agent, cells were incubated with $5 \mathrm{nmol} / \mathrm{l}$ TPL and a full range of concentrations of adriamycin. Results (Fig. 2D) revealed that $5 \mathrm{nmol} / \mathrm{l} \mathrm{TPL}$ increased the sensitivity of K562/A02 to adriamycin. Furthermore, the regulation of TPL on the cytotoxicity of adriamycin towards K562/A02 cells was also evaluated by quantification of the apoptotic cells. As shown in Fig. 2A, B and C, when adriamycin was combined with $5 \mathrm{nmol} / \mathrm{l} \mathrm{TPL}$, the mean apoptotic population of K562/ A02 cells was increased from 4.3 to $18.5 \%$, respectively, compared to $3 \mu \mathrm{g} / \mathrm{ml}$ of adriamycin treatment alone. The result suggested that the increased cytotoxicity on K562/A02 cells from the combination of TPL with adriamycin was achieved through enhancing the adriamycin-induced apoptosis.

Increased accumulation of adriamycin by TPL. The ability of TPL to increase the adriamycin-induced cytotoxicity towards K562/A02 cells was evaluated by intracellular adriamycinassociated mean fluorescence intensity (MFI). As shown in

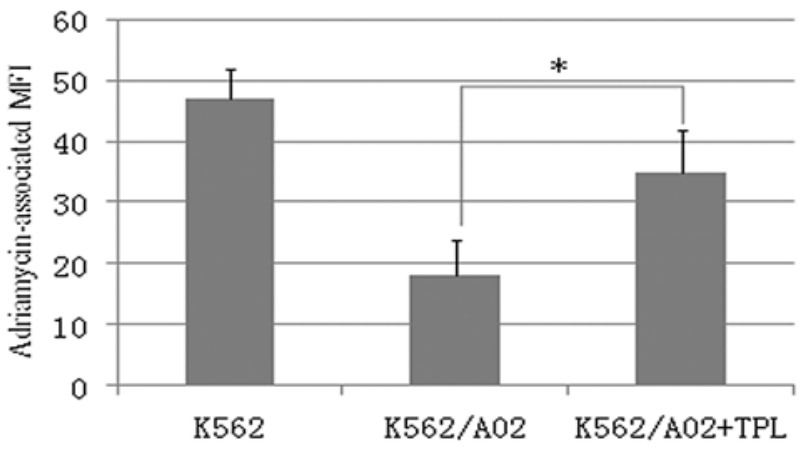

Figure 3. Effect of TPL on adriamycin accumulation in K562 and K562/A02 cells. Cells were treated with $3 \mu \mathrm{g} / \mathrm{ml}$ adriamycin in the absence and presence of TPL for $1 \mathrm{~h}$. Intracellular accumulation of adriamycin was evaluated by measuring the MFI, using flow cytometry. Data are expressed as the mean(s) \pm standard deviation of three independent experiments. $\mathrm{P}<0.05$ denotes statistically significant differences vs. untreated K562/A02 cells. MFI, mean fluorescence intensity; TPL, triptolide.

Fig. 3, the accumulation of adriamycin in K562/A02 cells was significantly lower than that in K562 cells. Following the treatment with $5 \mathrm{nmol} / 1 \mathrm{TPL}$ for $1 \mathrm{~h}$, respectively, intracellular accumulation of adriamycin was significantly increased in K562/A02 cells. TPL elevated the sensitivity of K562/A02 cells towards adriamycin by increasing adriamycin accumulation inside K562/A02 cells. The results provide strong evidence that TPL may effectively reverse P-glycoprotein-mediated MDR. 
A

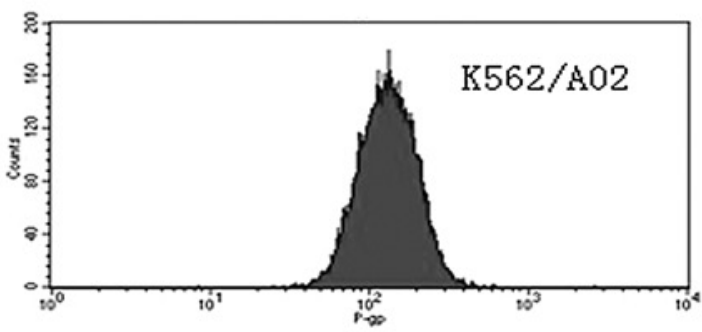

B

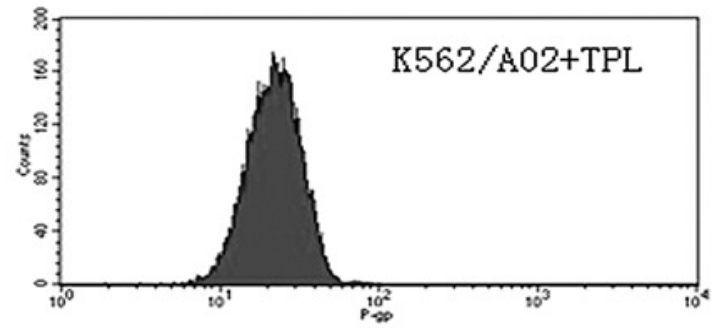

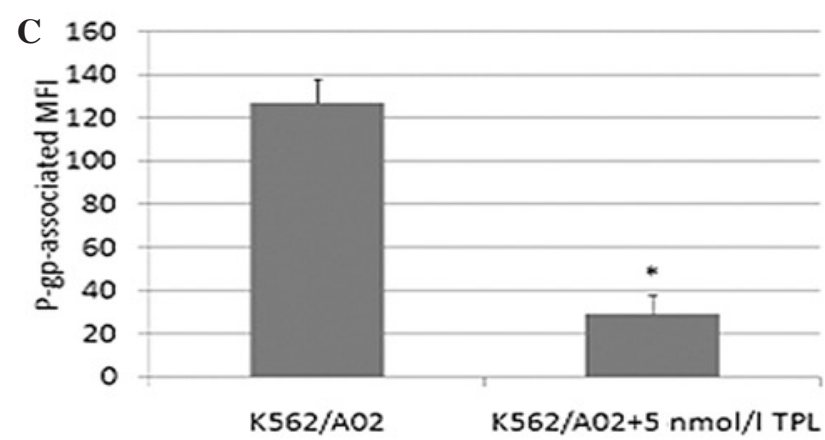

D

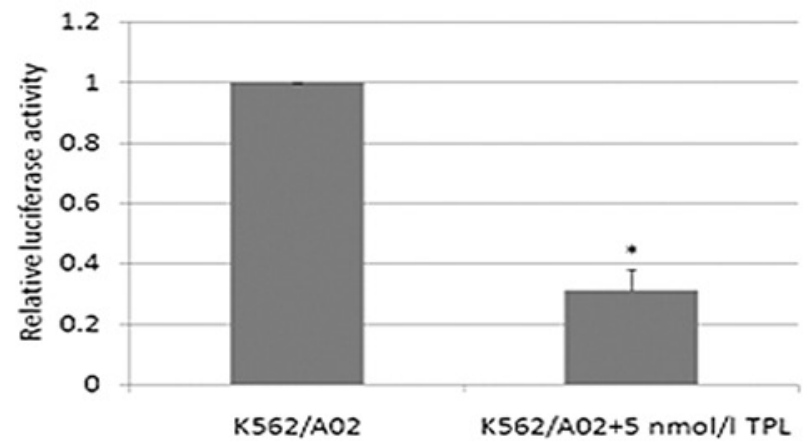

Figure 4. Inhibited expression of P-gp and MDR1 promoter activity by TPL. Cells were incubated with TPL for 24 h. P-gp was determined by flow cytometry using phycoerythrin (PE)-conjugated mouse anti-human $\mathrm{P}$-gp monoclonal antibody and the non-specific fluorescent labeling was corrected by the isotype control. MDR1 promoter activity was measured by flow cytometry. (A) Expression of P-gp in K562/A02 cells; (B) Expression of P-gp in K562/A02 cells treated with TPL; (C) P-gp-associated MFI; (D) MDR1 promoter activity. P-gp, P-glycoprotein; TPL, triptolide; MFI, mean fluorescence intensity.

Inhibited expression of P-glycoprotein and MDR1 promoter activity by TPL. To assess the effect of TPL on P-glycoprotein expression, flow cytometry was performed (Fig. 4A-C). A high level of P-glycoprotein expression was detected in K562/A02 cells. However, when K562/A02 cells were treated with TPL, the P-glycoprotein level was significantly decreased. MDR1 promoter activity was measured by the luciferase reporter gene assay (Fig. 4D). In the presence of TPL, MDR1 promoter activity was significantly decreased. The results indicate that TPL modulated the expression of P-glycoprotein through inhibition of the MDR1 promoter transcriptional activity.

\section{Discussion}

Chemotherapy is an indispensable tool used in cancer treatment. However, the emergence of cancer cell resistance to chemotherapy often hampers treatment efficacy. A major mechanism of resistance is the overexpression of drug-efflux pumps, such as P-glycoprotein (19). One strategy for the reversal of drug resistance in cells expressing $\mathrm{ABC}$ transporters is the combined use of anticancer drugs with modulators (20). Investigators have carried out numerous studies on drug resistance reversal in tumor cells in recent years and have developed 3 generations of drug resistance reversal agents, termed I, II and III (21), including verapamil, cyclosporin, quinidine and tamoxifen. These agents are capable of partly, or even completely, reversing drug resistance in in vitro experiments. Currently, however, most of these compounds have shown less than encouraging results. The aim of this study was to determine an effective and safe drug resistance reversing agent from Chinese traditional medicine, and to gain insight into its reversal effect and the molecular mechanism of that effect.

Previous studies have found that NF- $\mathrm{kB}$ is important in drug resistance. NF- $\kappa \mathrm{B}$ is a crucial transcription factor involved in multiple biological processes, including immune stress, cell differentiation, cell cycle control and cell apoptosis. Its functions in tumor formation, invasion, metastasis and drug resistance have captured increasing attention in recent years (22). Several studies have proven that MDR1 gene expression was regulated by NF- $\mathrm{KB}$. It was reported that NF- $\mathrm{KB}$ was capable of activating the transcription of reporter genes connected to the MDR1 promoter, suggesting that MDR 1 is a downstream gene regulated by NF- $\mathrm{kB}(1,23)$. Of note, a NF-кB-binding site was identified in the sequence between $-167 \mathrm{bp}$ and -158 bp upstream of the MDR1 gene (5'-GGGGAATTCC-3') by transient transfection technology. Furthermore, inhibition of NF- $\mathrm{kB}$ expression in HCT15 cells resulted in the downregulation of MDR1 mRNA/P-glycoprotein expression, indicating that NF- $\kappa \mathrm{B}$ plays a role in the regulation of drug resistance (24).

We became interested in TPL since it was reported that TPL is a potent inhibitor of NF- $\mathrm{kB}$ activation. Furthermore, the component monoterpenoids of the essential oil of Zanthoxyli fructus, demonstrate a strong inhibition of P-glycoprotein (25). TPL is a diterpenoid triepoxide derived from the herb Tripterygium wilfordii, and is proposed to have a similar molecular structure to the monoterpenoids. Therefore, we examined the effects of TPL on K562/A02 cells to observe whether this compound would show reversal of MDR activity. Moreover, we investigated the reversal effect of TPL on K562/A02 cells. 
A combination treatment of cells with TPL and adriamycin, revealed a significant increase of the sensitivity of adriamycin against K562/A02 cells. Expression of P-glycoprotein was markedly reduced in TPL treatment. In addition, the functional activity of P-glycoprotein to mediate drug efflux was decreased, leading to increased intracellular drug retention and drug resistance. TPL treatment combined with adriamycin was capable of promoting the adriamycin-mediated apoptosis of K562/A02 cells. These results demonstrate that TPL is capable of reversing the acquisition of drug resistance of K562/A02 cells. Recent results demonstrated the in vitro chemosensitizing effect of TPL on the cytotoxicity of 5-fluorouracil. Chen et al found TPL inhibited P-glycoprotein expression and enhanced the 5-fluorouracil antitumor effect on KB cells (26). In another study, TPL simultaneously induced reactive oxygen species, inhibited $\mathrm{NF}-\kappa \mathrm{B}$ activity and sensitized 5-fluorouracil in colorectal cancer cell lines (27). To the best of our our knowledge, this is the first study to explore the in vitro chemosensitizing effect of TPL on the cytotoxicity of adriamycin in leukemia cells.

In conclusion, TPL may effectively reverse MDR in K562/ A02 cells via inhibition of the expression and drug-transport function of P-glycoprotein.

\section{References}

1. Gottesman MM, Fojo T and Bates SE: Multidrug resistance in cancer: role of ATP-dependent transporters. Nat Rev Cancer 2: $48-58,2002$

2. Ushigome F, Takanaga H, Matsuo H, et al: Human placental transport of vinblastine, vincristine, digoxin and progesterone: contribution of P-glycoprotein. Eur J Pharmacol 408: 1-10, 2000

3. Marbeuf-Gueye C, Salerno M, Quidu P and Garnier-Suillerot A Inhibition of the P-glycoprotein- and multidrug resistance protein-mediated efflux of anthracyclines and calceinacetoxymethyl ester by PAK-104P. Eur J Pharmacol 391: 207-216, 2000.

4. Teodori E, Dei S, Martelli C, Scapecchi S and Gualtieri F: The functions and structure of ABC transporters: implications for the design of new inhibitors of Pgp and MRP1 to control multidrug resistance (MDR). Curr Drug Targets 7: 893-909, 2006.

5. Di Pietro A, Conseil G, Perez-Victoria JM, et al: Modulation by flavonoids of cell multidrug resistance mediated by P-glycoprotein and related ABC transporters. Cell Mol Life Sci 59: 307-322, 2002.

6. Chearwae W, Anuchapreeda S, Nandigama K, Ambudkar SV and Limtrakul P: Biochemical mechanism of modulation of human P-glycoprotein (ABCB1) by curcumin I, II, and III purified from Turmeric powder. Biochem Pharmacol 68: 2043-2052, 2004.

7. Kitagawa S: Inhibitory effects of polyphenols on p-glycoprotein-mediated transport. Biol Pharm Bull 29: 1-6, 2006.

8. Borowski E, Bontemps-Gracz MM and Piwkowska A: Strategies for overcoming $\mathrm{ABC}$-transporters-mediated multidrug resistance (MDR) of tumor cells. Acta Biochim Pol 52: 609-627, 2005.

9. Brinker AM, Ma J, Lipsky PE and Raskin I: Medicinal chemistry and pharmacology of genus Tripterygium (Celastraceae) Phytochemistry 68: 732-766, 2007.
10. Carter BZ, Mak DH, Schober WD, et al: Triptolide induces caspase-dependent cell death mediated via the mitochondrial pathway in leukemic cells. Blood 108: 630-637, 2006.

11. Yao GH, Luan JF, Ye D, et al: [Effects of triptolide on proliferation and apoptosis of Jurkat cell line in acute T lymphocytic leukemia]. Zhongguo Shi Yan Xue Ye Xue Za Zhi 16: 506-509, 2008 (In Chinese).

12. Lee KY, Chang W, Qiu D, Kao PN and Rosen GD: PG490 (triptolide) cooperates with tumor necrosis factor-alpha to induce apoptosis in tumor cells. J Biol Chem 274: 13451-13455, 1999.

13. Tang XY,Zhu YQ, Tao WH, Wei B and Lin XL: Synergistic effect of triptolide combined with 5-fluorouracil on colon carcinoma. Postgrad Med J 83: 338-343, 2007.

14. Chang WT, Kang JJ, Lee KY, et al: Triptolide and chemotherapy cooperate in tumor cell apoptosis. A role for the p53 pathway. J Biol Chem 276: 2221-2227, 2001.

15. Qu XJ, Yang JL, Russell PJ and Goldstein D: Changes in epidermal growth factor receptor expression in human bladder cancer cell lines following interferon-alpha treatment. J Urol 172: 733-738, 2004

16. Kim YK, Song YJ, Seo DW, et al: Reversal of multidrug resistance by 4-chloro-N-(3-((E)-3-(4-hydroxy-3-methoxy phenyl) acryloyl)phenyl)benzamide through the reversible inhibition of P-glycoprotein. Biochem Biophys Res Commun 355: 136-142, 2007.

17. Xu D, Lu Q and Hu X: Down-regulation of P-glycoprotein expression in MDR breast cancer cell MCF-7/ADR by honokiol. Cancer Lett 243: 274-280, 2006.

18. Okamoto T, Izumi H, Imamura T, et al: Direct interaction of $\mathrm{p} 53$ with the Y-box binding protein, YB-1: a mechanism for regulation of human gene expression. Oncogene 19: 6194-6202, 2000.

19. Calatozzolo C, Gelati M, Ciusani E, et al: Expression of drug resistance proteins Pgp, MRP1, MRP3, MRP5 and GST-pi in human glioma. J Neurooncol 74: 113-121, 2005.

20. Liscovitch M and Lavie Y: Cancer multidrug resistance: a review of recent drug discovery research. IDrugs 5: 349-355, 2002.

21. Perez-Tomas R: Multidrug resistance: retrospect and prospects in anti-cancer drug treatment. Curr Med Chem 13: 1859-1876, 2006.

22. Ramkissoon SH, Patel PS, Taborga M and Rameshwar P: Nuclear factor-kappaB is central to the expression of truncated neurokinin-1 receptor in breast cancer: implication for breast cancer cell quiescence within bone marrow stroma. Cancer Res 67: 1653-1659, 2007.

23. Amiri KI and Richmond A: Role of nuclear factor-kappa B in melanoma. Cancer Metastasis Rev 24: 301-313, 2005.

24. Bentires-Alj M, Barbu V, Fillet M, et al: NF-kappaB transcription factor induces drug resistance through MDR1 expression in cancer cells. Oncogene 22: 90-97, 2003.

25. Yoshida N, Takagi A, Kitazawa H, Kawakami J and Adachi I: Inhibition of P-glycoprotein-mediated transport by extracts of and monoterpenoids contained in Zanthoxyli fructus. Toxicol Appl Pharmacol 209: 167-173, 2005.

26. Chen YW, Lin GJ, Chuang YP, et al: Triptolide circumvents drug-resistant effect and enhances 5-fluorouracil antitumor effect on KB cells. Anticancer Drugs 21: 502-513, 2010.

27. Xu B, Guo X, Mathew S, et al: Triptolide simultaneously induces reactive oxygen species, inhibits NF-kappaB activity and sensitizes 5-fluorouracil in colorectal cancer cell lines. Cancer Lett 291: 200-208, 2010. 